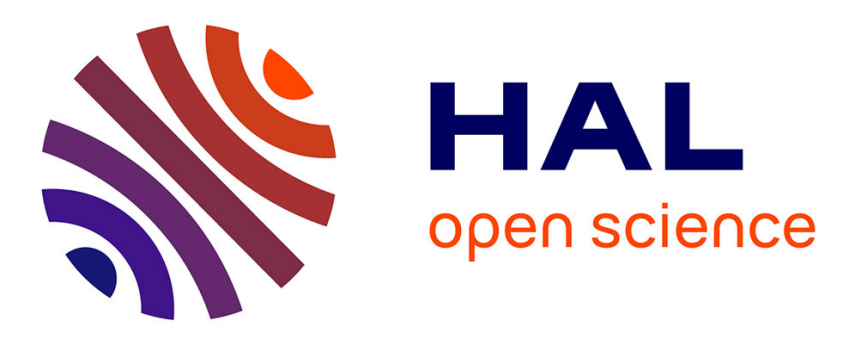

\title{
Static light scattering and electric birefringence experiments on saltfree solutions of poly(styrenesulfonate)
}

C. Johner, H. Kramer, S. Batzill, C. Graf, M. Hagenbüchle, C. Martin, R. Weber

\section{- To cite this version:}

C. Johner, H. Kramer, S. Batzill, C. Graf, M. Hagenbüchle, et al.. Static light scattering and electric birefringence experiments on saltfree solutions of poly(styrenesulfonate). Journal de Physique II, 1994, 4 (9), pp.1571-1584. 10.1051/jp2:1994218 . jpa-00248062

\section{HAL Id: jpa-00248062 https://hal.science/jpa-00248062}

Submitted on 1 Jan 1994

HAL is a multi-disciplinary open access archive for the deposit and dissemination of scientific research documents, whether they are published or not. The documents may come from teaching and research institutions in France or abroad, or from public or private research centers.
L'archive ouverte pluridisciplinaire HAL, est destinée au dépôt et à la diffusion de documents scientifiques de niveau recherche, publiés ou non, émanant des établissements d'enseignement et de recherche français ou étrangers, des laboratoires publics ou privés. 


\title{
Static light scattering and electric birefringence experiments on saltfree solutions of poly(styrenesulfonate)
}

\author{
C. Johner, H. Kramer, S. Batzill, C. Graf, M. Hagenbüchle, C. Martin and R. Weber \\ Fakultät für Physik, Universität Konstanz, 78434 Konstanz, Germany
}

(Received 8 March 1994, revised 2 May 1994, accepted 20 May 1994)

\begin{abstract}
Static light scattering and electric birefringence measurements on aqueous solutions of charged poly(styrenesulfonate) (PSS) with different molecular weights between $10^{5}$ and $1.1 \times 10^{6} \mathrm{~g} / \mathrm{mol}$ are presented. All experiments were performed in the dilute and the semidilute concentration regime $(0.0005 \mathrm{mg} / \mathrm{ml}<c<10 \mathrm{mg} / \mathrm{ml})$ at minimum ionic strength (down to $\approx 10^{-6} \mathrm{M}$ ). The static light scattering experiments show a single broad peak in the scattered intensity. The scattering vectors of these peaks increase with increasing concentrations $c$ and scale either with $c^{1 / 3}$ or with $c^{1 / 2}$ only depending on a relative concentration but not on the molecular weight : below about $20 c^{*}\left(1 c^{*}:=\right.$ overlap concentration of extended chains $=1$ particle/(contour length $\left.\ell_{c}\right)^{3}$ ) we found a $c^{1 / 2}$ dependence of the scattering vector, above $20 c^{*}$ a $c^{1 / 2}$ law is valid. A similar behaviour has been observed for rigid rods [1]. Our results are compared with previous light-[2, 3], small angle neutron-[4,5], and small angle $X$-ray [6] scattering investigations. Nearly all of these studies are in a very good agreement with the $c^{1 / 3}$-and $c^{1 / 2}$-law. The relaxation of the electric birefringence signal after an applied rectangular electric field is monoexponential in nearly all cases. Again the concentration $20 c^{*}$, independent of the molecular weight, seems to be a critical concentration : below $20 c^{*}$ we found a normal (negative) birefringence signal, above an anomal (positive) signal. These results are compared with results of electric birefringence measurements on aqueous solutions of rigid rods [7, 8] and PSS $^{-}$at different ionic strengths [911]. It is claimed that our results can be explained by a small flexibility of strongly elongated PSS $^{-}$-rods which increases slightly with increasing molecular weight and distinctly with raising concentration. The concentration $c^{*}$ is shown to be a reasonable quantity to describe polyelectrolyte solutions without added salt, independent of the molecular weight, respectively the contour length. By rescaling comparable data published by other authors we can establish the critical concentration $20 \mathrm{c}^{*}$, which seems to be universally valid.
\end{abstract}

\section{Introduction.}

Polyelectrolytes have been developed into one of the most examined objects in colloidal physics. While both the structure of solutions consisting of charged spheres [12] and the structure of solutions of rigid rods like tobacco mosaic viruses (TMV) or filamenteous DNA 
(fd) virus particles [1,3] are quite well investigated and theoretically understood, the structural properties of solutions of polyelectrolytes like poly(styrenesulfonate) are not still quite understood $[14,15]$. Soluted polymers at high ionic strength and/or high concentrations often show a high flexibility, they behave like Gaussian coils. Previous SANS [5] and SAXS [6, 16] investigations dealed with concentrations between $5 \mathrm{mg} / \mathrm{ml}$ and $300 \mathrm{mg} / \mathrm{ml}$. This regime is partially theoretically covered by Odjik [17], de Gennes [18] and Koyama [19] who calculated the persistence length and transition concentrations between different conformations of the polymers.

Our work deals with aqueous solutions of poly(styrenesulfonate) in the dilute/semidilute regime $(0.0005 \mathrm{mg} / \mathrm{ml}<c<10 \mathrm{mg} / \mathrm{ml})$. This polymer consists of a number $N$ of monomers each with a molecular weight of $206 \mathrm{~g} / \mathrm{mol}$ and a length of $0.25 \mathrm{~nm}$. The monomers are carrying an aromatic ring which leads to an intrinsic optical anisotropy of the polymer chain. In order to maximize the electrostatic interaction between the charged monomers of different polymers or monomers belonging to the same polymer, deionized («saltfree ») solutions are chosen. With a special experimental set-up we reach minimum ionic strengths down to about $10^{-6} \mathrm{M}$. Therefore the clouds of counterions surrounding the $\mathrm{PSS}^{-}$-particles extend up to several hundred nanometers. We shall see that this fact has crucial influence on the static and dynamic properties of PSS $^{-}$-solutions and distinguishes this paper from the work of Krämer et al. [9], Degiorgio et al. [10, 20] and Oppermann [11, 21].

Especially we are interested in the changes of the shape of the polyelectrolytes due to the flexibility of these chains which is determined by the contour length $\ell_{c}(=N \times$ monomer length), the particle concentration and the ionic strength. According to Odijk [17] we may introduce the persistence length

$$
\ell_{\mathrm{p}}=\ell_{1}+\ell_{\mathrm{e}}
$$

$\ell_{1}$ is the intrinsic part of the persistence length (1.2 nm for PSS) and $P_{\mathrm{e}}$ the electrostatic part being a function of the ionic strength. A strong electrostatic interaction between the monomers leads to a stiffening of the chain, respectively to an increase of the persistence length. An infinite persistence length means an infinitely rigid rod while Gaussian coils are represented by a vanishing persistence length.

Till now to our knowledge the only static light scattering data are the results of Drifford et al. $[3,22]$ being measured on samples of $7.8 \times 10^{5} \mathrm{~g} / \mathrm{mol}\left(\ell_{\mathrm{c}}=950 \mathrm{~nm}\right)$ and Krause et al. [2] on samples of $3.54 \times 10^{5} \mathrm{~g} / \mathrm{mol}\left(\ell_{\mathrm{c}}=430 \mathrm{~nm}\right)$ and $1.06 \times 10^{6} \mathrm{~g} / \mathrm{mol} \quad\left(\ell_{\mathrm{c}}=1.286 \mathrm{~nm}\right)$. Both data are in relative contradiction to each other. The SANS- and SAXS-measurements mentioned above dealed with molecular weights between $1.8 \times 10^{3}$ and $1.2 \times 10^{6} \mathrm{~g} / \mathrm{mol}$. The aim of our work is to extend the range of molecular weights in the light scattering regime from $10^{5} \mathrm{~g} / \mathrm{mol}\left(\ell_{\mathrm{c}}=121 \mathrm{~nm}\right)$ to $1.13 \times 10^{6} \mathrm{~g} / \mathrm{mol}\left(\ell_{\mathrm{c}}=1.374 \mathrm{~nm}\right)$ and to reconcile all scattering experiments. All these experiments show a peak in the scattered intensity which is caused by intermolecular electrostatic correlations. It is observed above and below the overlap concentration.

The solutions examined with the transient electric birefringence measurements $(0.0005 \mathrm{mg} / \mathrm{ml} \leqslant c \leqslant 10 \mathrm{mg} / \mathrm{ml})$ are optically isotropic if no external electric field is applied, that means the particles are isotropically oriented. However at low concentrations, when no interparticle interaction occurs, it is easy to orient the polyelectrolytes in the direction of an applied electric field, the solutions become optically anisotropic and therefore birefringent. The sign of the birefringence signal is determined by the aromatic groups, it is negative and called normal. After switching off the applied electric field the particles relax to an isotropic orientation and the signal vanishes. There are some theories both for rigid rods and for flexible chains calculating the time constant $\tau$ of this decay of the birefringence signal and calculating 
the related rotational diffusion constant $D_{\mathrm{R}}$ as well. In the case of a monoexponential decay $D_{\mathrm{R}}$ can be deduced by using the formula

$$
\begin{gathered}
\Delta n(t)=\Delta n_{0} \exp (-t / \tau) \\
D_{\mathrm{R}}=1 /(6 \tau)
\end{gathered}
$$

where $\Delta n(t)$ is the decay of the electric birefringence and $\Delta n_{0}$ is the steady state value [7]. The dependence of $D_{\mathrm{R}}$ on the steric particle parameters is given by Newman et al. [23], and quite recently the validity has been again confirmed experimentally [7]

$$
D_{\mathrm{R}, \text { rod }}=\frac{3 \cdot k_{\mathrm{B}} \cdot T}{\pi \cdot \eta \cdot L^{3}} \cdot\left[\ln \left(\frac{2 L}{d}\right)-1.45+7.5 \cdot\left\{\frac{1}{\ln \left(2 \cdot \frac{L}{d}\right)}-0.27\right\}^{2}\right]
$$

where $k_{\mathrm{B}} T$ is the thermal energy, $\eta$ is the solvent viscosity, $L$ and $d$ are the length and the diameter of the rod. The relation for $\tau$ for flexible polymers is calculated by Yoshizaki $e t$ al. [24]:

$$
\tau(x)=\frac{1}{6 \cdot D_{\mathrm{R}, \text { rod }}} \cdot\left[x+\frac{\mathrm{e}^{-21}-1}{2}\right]^{15} \frac{[1+0.539526 \cdot \ln (1+x)]}{x^{3}}
$$

where $x=\ell_{c} / 2 \ell_{p}$. This equation enables the estimation of the persistence length of our polymers : in case of $x \ll 1$ the rigid rod limit is reached, in case of $x \approx 1$ we have distinct flexible polyelectrolytes, and in case of $x \gg 1$ the polymers can be considered as Gaussian coils.

Above a critical particle concentration the sign of the birefringence signal changes from negative to positive. This behaviour, called the anomaly of the electric birefringence, has been already observed in aqueous solutions of rigid rods like charged TMV [25], charged fd viruses $[8,26]$ and micelles [27] and of charged PSS ${ }^{-}$rods $[9,21]$ as well. There are several models trying to explain the anomaly $[27,28]$. To our opinion the following idea seems to be most successful (a more detailed description is given in Ref. [8]) : the orientation of rods in an electric field is determined by the whole system minimizing its free energy which is the sum of different parts [29]. The first one is determined by the interaction of a single rod with the electric field. The second one describes the electrostatic particle-particle interaction while the third one expresses the orientational entropy. The fourth one is only an unimportant constant. In the case of negligible electrostatic particle-particle interaction (for example in very dilute solutions) and/or at strong electric fields the first term will dominate and the particles are oriented in direction of the external field. But at somewhat higher concentrations and low electric fields the particle-particle interaction part dominates, and therefore a perpendicular orientation of the particles towards the external field is favoured, which is proved by static light scattering experiments on aqueous solutions of fd virus particles in an electric field [26]. This interaction takes especially effect at low electric fields and very low ionic strengths, respectively at very large Debye screening lengths $\kappa^{-1}$ [7]. In this paper results at small electric fields $\left(4 \times 10^{2} \mathrm{~V} / \mathrm{m} \leqslant E \leqslant 3 \times 10^{4} \mathrm{~V} / \mathrm{m}\right)$ are presented.

Above a second critical concentration another change of the birefringence signal from positive back to negative occurs [9]. Krämer et al. assume that the particles, building up a three-dimensional network, are oriented in direction of the electric field again.

Therefore it is useful to define different concentration regimes. Analogous to the definition of the overlap concentration of extended rods $1 c^{*}$, at which sterical interaction of extended rods becomes possible, Kaji et al. [6] suggest an overlap concentration $1 c_{\mathrm{s}}^{*}$, using the radius 
of gyration $\left\langle S^{2}\right\rangle$ :

$$
\begin{gathered}
c_{\mathrm{s}}^{*}=\frac{N}{\frac{4}{3} \cdot \pi \cdot\left\langle S^{2}\right\rangle^{3 / 2}} \\
\left\langle S^{2}\right\rangle=\ell_{\mathrm{p}}^{2}\left[\frac{\alpha}{3}-1+\frac{2}{\alpha^{2}} \cdot\left(\alpha-1+\mathrm{e}^{-\alpha}\right)\right]
\end{gathered}
$$

where $\alpha=\ell_{\mathrm{c}} / \ell_{\mathrm{p}}$. With decreasing concentration the peak indicating a liquid like phase disappears, the intermolecular distances are getting larger than the electrostatic screening length $\kappa^{-1}$, the intermolecular correlations are lost and a gas like phase is obtained. This crossover concentration $c_{\kappa}^{*}$ can be estimated [6] as

$$
c_{\kappa}^{*}=\frac{1}{4 \cdot \pi^{3} a^{3} N^{2}}
$$

here $a$ is the length of a monomeric unit being equal to $0.25 \mathrm{~nm}$.

Finally Odijk [17] calculated the concentration $c^{* *}$ at which the polyelectrolyte lattice melts :

$$
c^{* *}=\frac{0.04}{4 \cdot \pi \cdot Q^{2} a} \approx 8.9 \mathrm{mg} / \mathrm{ml}
$$

where $Q=\frac{e^{2}}{4 \cdot \pi \cdot \varepsilon_{0} \cdot \varepsilon \cdot k_{\mathrm{B}} \cdot T} \approx 0.7 \mathrm{~nm}$ is the Bjerrum length in water $\left(T=20^{\circ} \mathrm{C}\right)$ and $\varepsilon_{0}$ ist the permittivity of vacuum and $\varepsilon$ the relative permittivity. Of course all samples measured in our experiments are still liquid like. The calculated concentration $c^{* *}$ seems to be not valid. In order to avoid any confusion we will only use absolute concentrations in units of $\mathrm{mg} / \mathrm{ml}$ and relative concentrations in units of $c^{*}$

\section{Experiment.}

The sodium salt of poly(styrenesulfonate) (NaPSS) supplied by polyscience is according to the manufacturer characterized by $M_{\mathrm{w}} / M_{\mathrm{n}} \leqslant 1.1$ and a degree of sulfonation $>90 \%$. It was used without further purification. In order to obtain polyelectrolytes with various contour lengths $\ell_{\mathrm{c}}$ we used five different molecular weights : MW100 $=1.0 \times 10^{5} \mathrm{~g} / \mathrm{mol} \quad\left(\ell_{\mathrm{c}}=121 \mathrm{~nm}\right)$, MW200 $=2.0 \times 10^{5} \mathrm{~g} / \mathrm{mol} \quad\left(\ell_{\mathrm{c}}=243 \mathrm{~nm}\right), \quad$ MW400 $=4.0 \times 10^{5} \mathrm{~g} / \mathrm{mol} \quad\left(\ell_{\mathrm{c}}=485 \mathrm{~nm}\right)$, MW780 $=7.8 \times 10^{5} \mathrm{~g} / \mathrm{mol} \quad\left(\ell_{\mathrm{c}}=946 \mathrm{~nm}\right) \quad$ and $\quad \mathrm{MW1} 132=1.132 \times 10^{6} \mathrm{~g} / \mathrm{mol}$ $\left(\ell_{\mathrm{c}}=1373 \mathrm{~nm}\right)$. First, for each molecular weight a stock solution of either $1 \mathrm{mg} / \mathrm{ml}$.or $10 \mathrm{mg} / \mathrm{ml}$ was prepared by dissolving the carefully weighted salt in deionized water $(R>18 \mathrm{M} \Omega$ ), then the solution was checked by an absorption measurement. The absorption coefficient (measured with a Beckmann spectrometer DU 64, Darmstadt, Germany) at the absorption maximum $(\lambda=224 \mathrm{~nm})$ was in a good agreement with that of other authors [ 30 , 31]. The desired concentrations were obtained by dilution.

The light scattering apparatus is a commercial instrument (ALV, Langen, Germany) consisting of a computer controlled goniometer table with focusing and detector optics, a power stabilised $3 \mathrm{~W}$ argon ion laser (Spectra Physics), a digital rate meter and a temperature control which stabilises the temperature of the sample cell at $20 \pm 0.1^{\circ} \mathrm{C}$. The available scattering vector $q=\frac{4 \cdot \pi \cdot n}{\lambda_{0}} \cdot \sin \left(\frac{\vartheta}{2}\right)$ ranges from $0.01 \mathrm{~nm}^{-1} \leqslant q \leqslant 0.033 \mathrm{~nm}^{-1}$ where 
$\lambda_{0}=488 \mathrm{~nm}$ is the vacuum wavelength of the incident beam, $n=1.33$ the refractive index of the solution and $\vartheta$ the scattering angle. The intensities were measured in steps of $5^{\circ}$ and normalized to a reference sample (toluene) to correct power fluctuations and to get a standard of the incident laser intensity. Furthermore, the background scattering due to water and the dark rate of the photomultiplier were subtracted from the measured count rates. Also the usual correction due to the geometrical scattering volume was made. In order to avoid any dirt of dust all vessels, for example the light scattering cells, were cleaned with acetone following ethanol and finally excessively with highly purified water. Every step of preparation occurred in a dust free flow box. In addition all samples were centrifuged at $6000 \mathrm{rpm}$ for at least $3 \mathrm{~h}$ before each measurement. In order to reach the minimum ionic strength, a cleaned mixed-bed ion exchange resin (Serva Diagnostics; Heidelberg, FRG, lot No. 45 500) but no neutralising agent like $\mathrm{NaOH}$ was added to each sample. In good approximation no small ions except of $\mathrm{H}^{+}$and $\mathrm{OH}^{-}$ are left in the solution. Therefore the minimum ionic strength only depends on the particle concentration.

The used birefringence apparatus is a commercial instrument (spectrometer DB 10, Suck, Siegen, FRG) and very similar to that described elsewhere [32]. We measured at a wavelength of $\lambda=632.8 \mathrm{~nm}$ and at a temperature of $20.0 \pm 0.1{ }^{\circ} \mathrm{C}$. The pulse lengths were chosen in a way that the maximum of the birefringence signal was reached. It was proved that the measurements were performed in the Kerr regime, respectively at the border of the Kerr regime in the case of the highest molecular weight.

Again we wanted to reach minimum ionic strength wherefore a tube pump system was used $[33,34]$. For that purpose the solution was pumped through this closed circuit, which includes the birefringence cell and the mixed-bed-ion exchange resin. Always the conductivity was measured in order to proof that the minimum ionic strength was reached.

\section{Results and discussion.}

Figure 1 shows the normalized intensity $I(q)$ of MW1132 at concentrations between $0.015 \mathrm{mg} / \mathrm{ml}$ and $0.050 \mathrm{mg} / \mathrm{ml}$ obtained by static light scattering.

The intensities of all molecular weights and measured concentrations $c$ exhibit a single broad but well defined peak at certain $q_{\mathrm{m}}$. In addition $I(q)$ increases with decreasing $q$ as observed previously $[2,3]$. The peaks for poly(styrenesulfonate) with $\mathrm{MW} 100=10^{5} \mathrm{~g} / \mathrm{mol}$ were hardly detectable because of a very low scattering intensity.

In figure 2 the position of the peak maxima for all molecular weights are plotted versus concentration. Very similar to the results found by Krause et al. [2] a gap between the peak positions of the higher $\left(\mathrm{MW} \geqslant 7.8 \times 10^{5} \mathrm{~g} / \mathrm{mol}\right)$ and the lower $\left(\mathrm{MW} \leqslant 4.0 \times 10^{5} \mathrm{~g} / \mathrm{mol}\right)$ molecular weights appears. The data obtained by Drifford et al. [3] $\left(\mathrm{MW}=7.8 \times 10^{5} \mathrm{~g} / \mathrm{mol}\right)$ lie somewhat below ours. The positions of the maxima scale with certain power laws : for the lower molecular weights we found $q_{\mathrm{m}} \propto c^{1 / 3}$, for the higher ones the exponents increase pointing to a «non-Gaussian » shape of our polyelectrolytes : it would be expected that in the dilute/semidilute regime Gaussian coils behave very similar to spheres with a well known $c^{1 / 3}$-dependence of the scattering vector of the peak maximum. The exact values of our exponents are listed in table $\mathbf{I}$.

Koyama et al. [19] predict for very high $c \times N$ values

$$
q_{\mathrm{m}} \propto\left(\frac{c}{N}\right)^{\frac{1}{2}}
$$




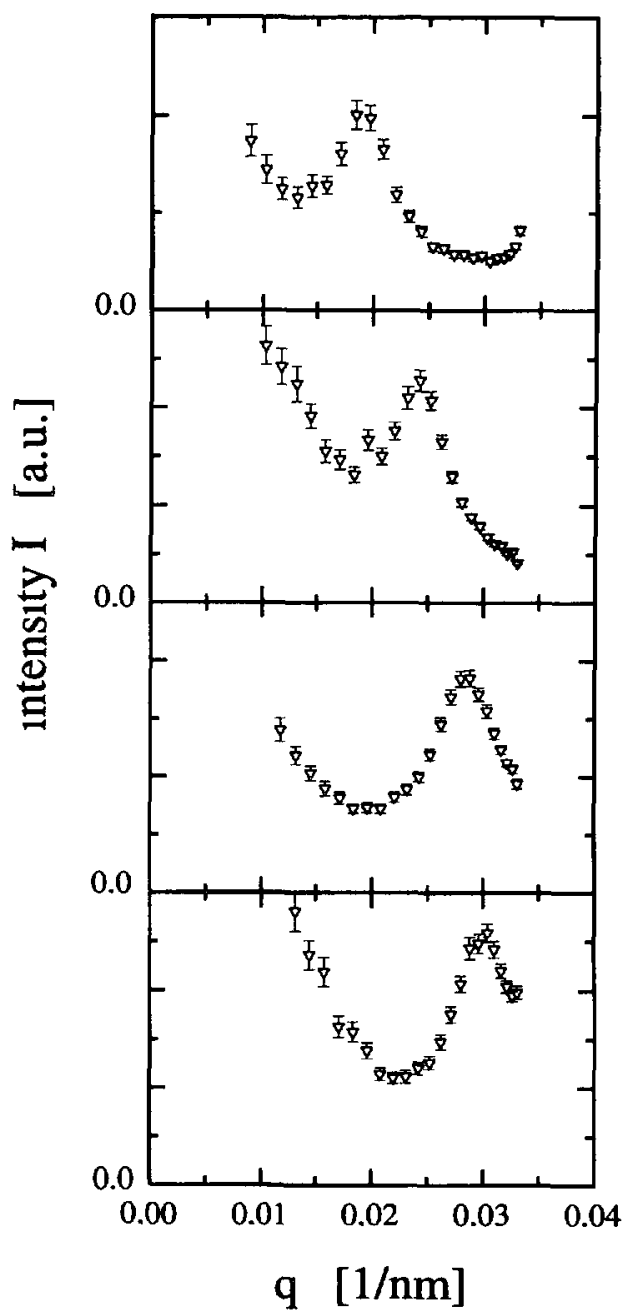

Fig. 1. - Intensities of four samples with molecular weight $M W 1132=1.132 \times 10^{6} \mathrm{~g} / \mathrm{mol}$ : the concentrations are (starting from the top) : $c=0.015 \mathrm{mg} / \mathrm{ml}, c=0.031 \mathrm{mg} / \mathrm{ml}, c=0.040 \mathrm{mg} / \mathrm{ml}$, $c=0.050 \mathrm{mg} / \mathrm{ml}$.

and for very small $c \times N$ values (dilute solutions)

$$
q_{\mathrm{m}} \propto(c)^{\frac{1}{3}}
$$

which are obtained for particles reduced to their centre of mass. The molecular weights MW 100, MW200, and MW400 fulfil the condition of the latter case and are in agreement with Koyamas prediction. The higher ones (MW780 and MW1132) seem to be in the transition regime between these two extrema. This might explain differences between our results and the results obtained by Drifford and Krause.

The relaxation times of the electric birefringence are plotted in figure 3 versus the particle concentration for MW400 and MW1132. In the dilute regime the electric birefringence signal 


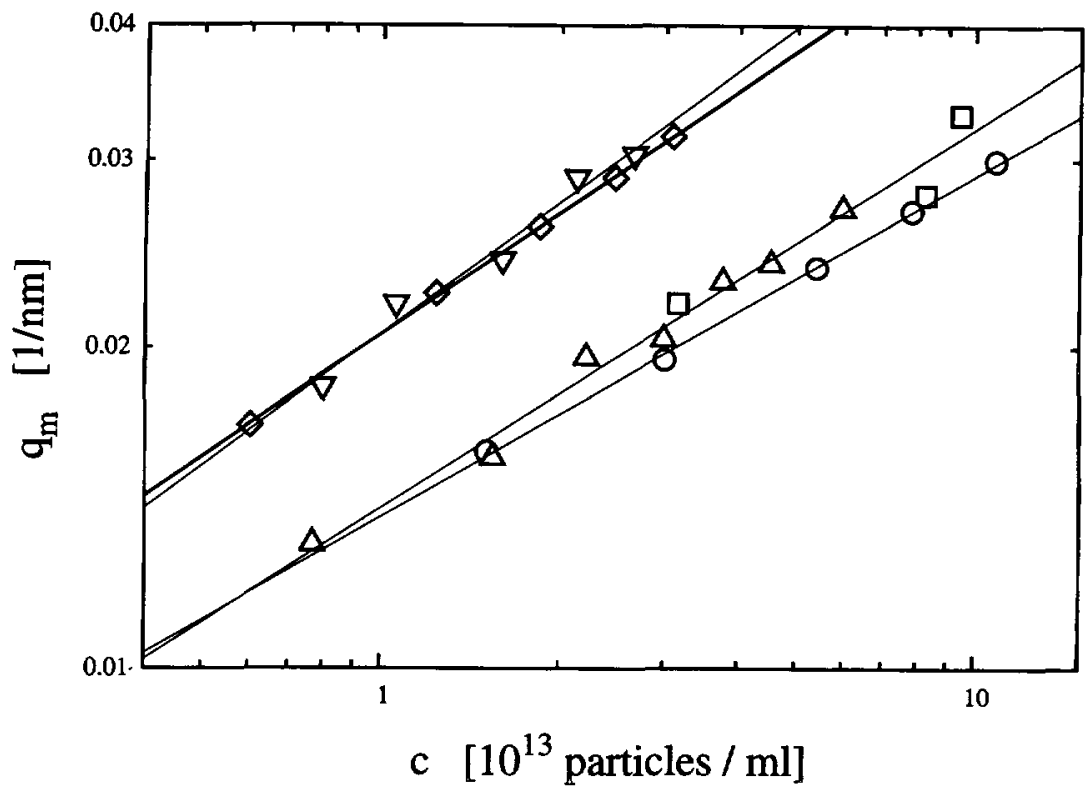

Fig. 2. - Positions of the maxima of the scattered intensities $q_{\mathrm{m}}$ of the samples MW100 ( $\square$ ), MW200 $(0)$, MW400 $(\triangle)$, MW780 $(\diamond)$ and MW1132 $(\nabla)$ in dependence of the concentration are shown in a double logarithmic scale. Also the accompanying fits are plotted. The results of these fits are listed in table I.

Table I. - The results from a least square fit of $q_{\mathrm{m}}(c)$ are listed for all used molecular weights. (See Fig. 2).

\begin{tabular}{|c|c|c|}
\hline \multirow{2}{*}{ Molecular weight MW [g/mol] } & \multicolumn{2}{|c|}{ Linear fit for $\ln \left(q_{\mathrm{m}}(c)\right)=A+B \cdot \ln (c)$} \\
\cline { 2 - 3 } & $A$ & $B$ \\
\hline 100,000 & -1.80 & $0.29 \pm 0.11$ \\
\hline 200,000 & -1.89 & $0.32 \pm 0.02$ \\
\hline 400,000 & -1.85 & $0.35 \pm 0.02$ \\
\hline 780,000 & -1.69 & $0.38 \pm 0.04$ \\
\hline $1,132,000$ & -1.69 & $0.41 \pm 0.04$ \\
\hline
\end{tabular}

of the shorter chains (MW400 and MW200, in the case of the latter one we made only exemplary examinations) decays monoexponentially while the decay of the birefringence signal of the long PSS $^{-}$particles can be fitted with two relaxation times. Typical representatives of these two signal types and the belonging fits are plotted in figure $4 \mathrm{~A}$ and $\mathrm{B}$. As expected the shorter chains relax much faster than the longer ones. In the very dilute regime $\left(c \leqslant 5 \times 10^{-4} \mathrm{mg} / \mathrm{ml}\right.$ ) the relaxation times (in the case of MW1132 we are talking of the 


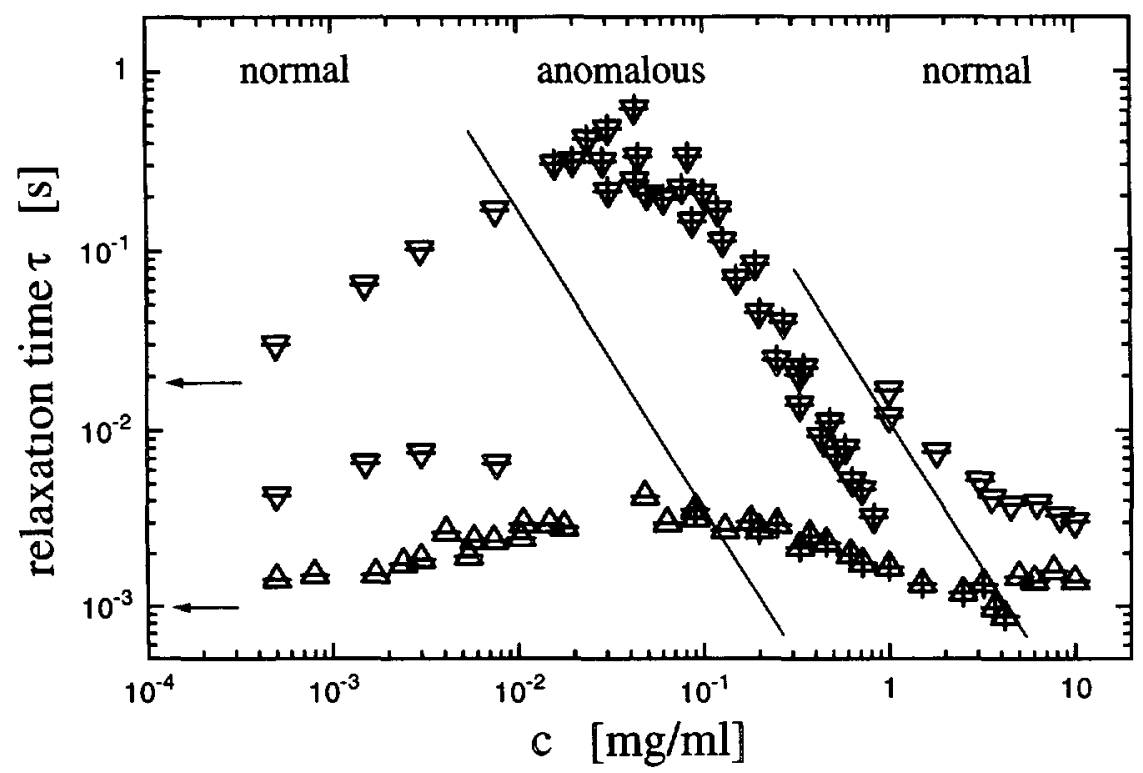

Fig. 3. - The relaxation times of the birefringence signal as a function of the PSS concentration. The symbols are the same as defined in figure 2 . The « plus », respectively the « minus » symbols indicate the sign of the birefringence signals. The arrows indicate the calculated values (using Eqs. (2a), (2b) and (3)) for rigid rods with a length being the same as the contour length of MW 1132 (upper arrow). respectively the contour length of MW400 (lower arrow). The two solid lines separate concentration regimes with apposite signs of the birefringence signal.

(dominating) larger times) are about the same as for rigid extended rods calculated by equation (3). With increasing concentration the relaxation is increasingly hindered. This behaviour is well-known from rigid rods $[7,34]$ and plotted for fd virus solutions in figure 5B. Above a first critical concentration the relaxation becomes faster and the sign of the electric birefringence changes from negative (normal signal) (Fig. 4A and $B$ ) to positive (anomalous signal) (Fig. 4C). A second critical concentration can be observed where the sign changes back to negative (Fig. 4D) while the relaxation of the birefringence signal remains nearly unchanged. Except of very small transition regimes the signals are always purely either positive or negative.

The interparticle interaction has crucial influence on the relaxation times. As shown for rigid rods [7] the relaxation of oriented particles is with increasing concentration increasingly hindered. At low concentrations PSS particles behave very similar pointing out their stretched character. But with increasing concentration and therefore increasing interparticle interaction the relaxation seems to be less hindered pointing out a starting coiling of the PSS rods. All relaxation processes are rotations of single (but interacting) particles and not due to a rotation for example of clusters. It is not conceivable that a rotation of clusters could give a monoexponential decay and could be faster than that of a single and not interacting rod. Apart from this fact one should mention that a suspension consisting of these clusters would remain optically birefringent (!) which is not the case.

There are some other electric birefringence results on PSS - available. Degiorgio et al. [20] examined dilute solutions of short PSS ${ }^{-}$chains (MW $\leqslant 2 \times 10^{5} \mathrm{~g} / \mathrm{mol}$ ) and noticed monoexponential decays, too. Their relaxation times are somewhat below ours. This can be explained by a larger ionic strength which is (at the same particle concentration) about fifteen times the 

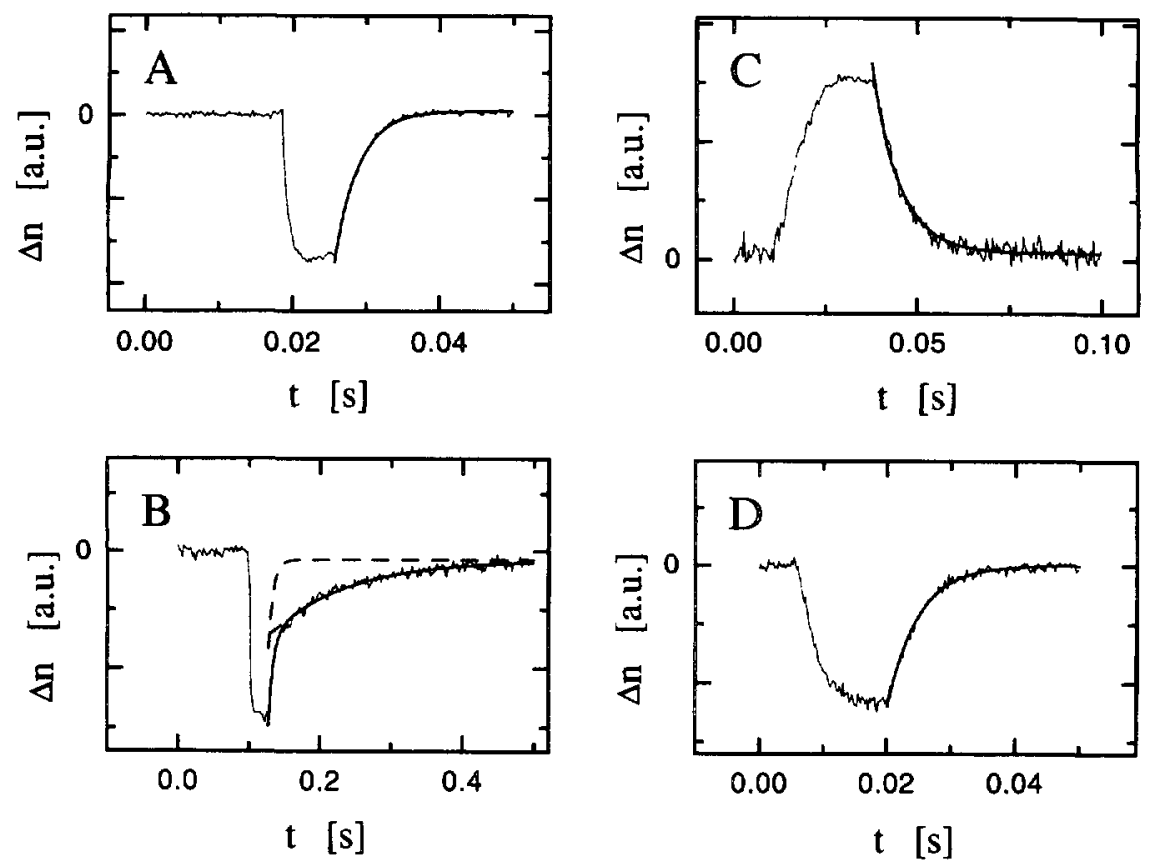

Fig. 4. - Typical birefringence signals in different concentration regimes fitted with a one exponential decay in A, C, D (full drawn line) : A) Normal birefringence signal of MW400 with a concentration $c=0.090 \mathrm{mg} / \mathrm{ml}\left(E=2.8 \times 10^{4} \mathrm{~V} / \mathrm{m}\right)$. B $)$ Normal birefringence signal of MW1132 with a concentration $c=0.008 \mathrm{mg} / \mathrm{ml}\left(E=2.8 \times 10^{4} \mathrm{~V} / \mathrm{m}\right)$. The decay is fitted with two one exponential decay curves (dashed lines). The sum of both curves is plotted as a solid line. C) Anomalous birefringence signal of MW 1132 with a concentration of $c=0.520 \mathrm{mg} / \mathrm{ml}\left(E=3.2 \times 10^{3} \mathrm{~V} / \mathrm{m}\right)$. D) Normal birefringence signal of MW1132 with a concentration of $c=4.38 \mathrm{mg} / \mathrm{ml}\left(E=4.0 \times 10^{4} \mathrm{~V} / \mathrm{m}\right)$.

ionic strength used in our experiment. A higher ionic strength causes smaller clouds of counterions and therefore a smaller particle-particle interaction, that means the relaxation is less hindered.

The relaxation processes measured by Krämer et al. [9] and Oppermann [35] on samples of different molecular weights do not show in the dilute regime an increase of the relaxation times with increasing concentration, and they are too fast to be only explained by different ionic strengths. Oppermann himself solved this discrepancy in a later publication [21]: too strong applied electric DC (direct current) fields start to strip off the ion cloud surrounding the particles, and the linear charge density becomes a function of the position along the rod. The persistence length, however, describing the stiffness of rods is a function of this charge density. For that reason the particles start a local coiling. In this later publication on low concentrations [21] Oppermann avoided these «artefacts» by using the dynamic electric birefringence technique (alternating current (AC) fields) and obtained the same results as we get by using very small field strengths. We assume that the data published by Krämer et al. [9] suffer from to high electric DC fields, too, which are up to two orders of magnitude stronger then ours. A detailed study of effects of strong electric fields on the birefringence signal is in progress. As a first conclusion we should mention that :

1) At low particle concentrations the decays of the birefringence signals of MW400 are monoexponential, and the relaxation times are comparable to that of rigid rods wherefore the 
particles have to be considered as relatively stiff and nearly stretched. The decay of the birefringence of MW1132 is not quite monoexponential indicating a small influence of bending modes. As plotted in figure $5 \mathrm{~B}$ the dominating (slower) relaxation times of suspensions of MW1132 $\left(\ell_{c}=1373 \mathrm{~nm}\right)$ are comparable to those of suspensions of fd viruses (which are rigid and about $900 \mathrm{~nm}$ long) and pointing out nearly stretched rods. Our results are in a good agreement with those of other authors [21,9] and theoretical computations by Odijk et al. [17] who calculated the persistence length as follows:

$$
\begin{array}{ll}
\ell_{\mathrm{p}}=\frac{1}{4 \cdot Q \cdot \kappa^{2}}+\ell_{1} ; \quad \sigma \leqslant Q \\
\ell_{\mathrm{p}}=\frac{Q}{4 \cdot \kappa^{2} \sigma^{2}}+\ell_{\mathrm{i}} \quad \sigma>Q
\end{array}
$$

where $\sigma$ is the mean distance of two neighboured charges assumed to be about the length of a monomeric unit $a=0.25 \mathrm{~nm}$. Therefore equation (9a) has to be taken in order to calculate the persistence lengths of PSS ${ }^{-}$. In the case of no salt (minimum ionic strength) we obtain for the lower concentration regime for MW400 (as an example) a persistence length which is about ten times larger than the contour length, that means these rods are rigid and stretched. On the other hand for MW1132 a persistence length which is about the same as the contour length is found, in this case the PSS $^{-}$molecule is bent.

Stevens and Kremer [36], who performed Monte-Carlo (MC)-simulations on solutions of charged but relatively short chains, find even for very dilute concentrations chains which are not rigid and not quite stretched. They calculate strong fluctuations of the shape of the particles "leading to horseshoe-shaped configurations ». Our results contradict such a behaviour. But their conception of a « very dilute » concentration differs from our definition : they consider experiments examining the configuration of particles at concentrations of $9 \times 10^{-5} \mathrm{monomol} / \mathrm{l}$ $\left(=1.85 \times 10^{-2} \mathrm{mg} / \mathrm{ml}\right.$ PSS $)$ to be not physically realizable.

2) At higher particle concentrations a strong particle-particle interaction occurs. This leads as discussed above, to an orientation of the particles perpendicular to the electric field. Furthermore we observe with increasing concentration decreasing relaxation times. Equation (3) connects the relaxation times with lengths $L$. The quantity $\left(\frac{\tau}{\tau_{0}}\right)^{1 / 3}$ which is about proportional to this apparent length $L$ divided by $\ell_{c}$ decreases to values somewhat below one indicating a slight coiling. The quantity $\tau_{0}$ is the theoretically calculated relaxation time of a rigid and extended rod with a length being equal to $\ell_{c}$. Nevertheless they decay of the birefringence signal remains exactly monoexponential. The particles seem to be rather rigid but no more quite elongated.

3) Raising the particle concentration changes the sign of the birefringence signal once again. Again we find a monoexponential decay. The relaxation times depend only weakly on concentration : above about $1000 c^{*}$ the average particle-particle distance is about the distance between two neighboured monomers belonging to the same rod. Therefore the ionized groups of the PSS ${ }^{-}$chains can be considered in a good approximation to be distributed uniformly in the solution, and the Debye screening length $\kappa^{-1}$ is proportional to $c^{-1 / 2}$ [37]. The static light scattering experiments indicate a dependence of the typical monomer-monomer distance $r$ as $c^{-1 / 2}$, too. The interaction, which is a function of $\kappa \cdot r$, does not depend any longer on the concentration.

In figure $5 \mathrm{~A}$ our and results of other authors from light scattering [2, 3], SANS [4, 14] and SAXS [6] experiments performed on PSS $^{-}$are « rescaled» by plotting $q_{\mathrm{m}} \ell_{\mathrm{c}}$ versus $c^{*}$ (A) and compared with the results obtained by electric birefringence measurements (B), also plotted versus $c^{*}$ 


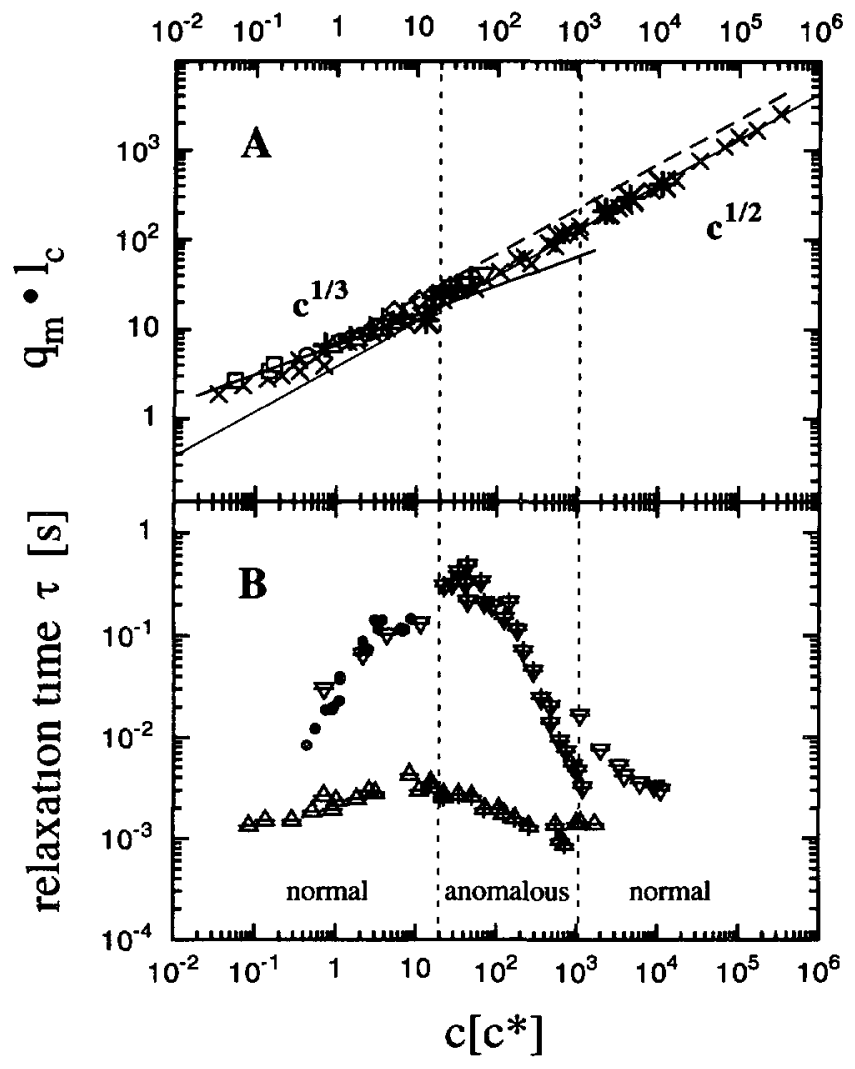

Fig. 5. - The symbols are the same as in figures 2 and $3:$ A) the wavevectors of the maximum of the scattered intensities times the contour length $\ell_{c}$ are plotted versus the concentration in units of $c^{*}$. Additionally the results of light scattering $[2,3](+)$, SANS $[4,14]$ and SAXS $[6]$ measurements on PSS (X) and SANS measurements on PVP [15] (*) are shown. The dashed line is theoretically predicted in reference [1] for rigid rods, the full lines are calculated for polyelectrolyte solutions by Koyama [19] (Eqs. (8a) and (8b)). B) The results shown in figure 3 are plotted versus concentration in units of $c^{*}$, too, and are compared with the results on fd virus particles $(\bullet)$.

Part A of figure 5 shows obviously that equations (8a) and (8b) taken together are generally valid over seven (!) orders of magnitude, and that there is no dependence on the contour length $\ell_{c}$ respectively the molecular weight or the absolute concentration (for example in $\mathrm{mg} / \mathrm{ml}$ ) recognizable. All samples have a critical concentration _ expressed in units of $c^{*} \ldots$ in common. Below this the 1/3-exponent is found, above the $1 / 2$-exponent is valid. (Also SANS measurements [15] on Poly(vinylpyridine) fulfil these laws.) These facts are comparable to the behaviour of rigid (and stretched) rods like TMV or fd; the power laws obtained by scattering experiments and theoretically computed [1] are shown in figure 5A. The $c^{1 / 2}$ law is plotted as a dashed line. The $c^{1 / 3}$ laws of rigid rods and polyelectrolyte solutions (Eq. ( $\left.8 \mathrm{~b}\right)$ ) are the same and are plotted as a solid line. Assuming an effective length $\ell_{\text {eff }}$ allows us to attribute our $q_{\mathrm{m}} \ell_{\mathrm{c}}$ values to the already known «rigid rod values", that means in static scattering experiments the polyelectrolytes with a concentration above $20 c^{*}$ behave like rigid rods with new lengths $\ell_{\text {eff }} \approx 1 / 3 \cdot \ell_{\mathrm{c}}$. This « rescaling» was achieved by replacing the quantities $q_{\mathrm{m}} \ell_{\mathrm{c}}$ by $q_{\mathrm{m}} \ell_{\text {eff }}$ and $c^{*}=1$ particle $/ \ell_{\mathrm{c}}^{3}$ by $c^{*}=1$ particle $/ \ell_{\mathrm{eff}}^{3}$. This exchange of $\ell_{\mathrm{c}}$ for $\ell_{\text {eff }}$ shifts the new $c^{1 / 2}$ line (plotted in Fig. 5A as a solid line) back to the "rigid rod" 
$c^{1 / 2}$ line (dashed line). The length $\ell_{\text {eff }}$ is very similar to the length $L$ introduced above which is obtained by the birefringence experiments. In good agreement with the results from the electric birefringence in the dilute regime the short PSS- chains (MW100 and MW200) seem to be stretched while the longer chains in the dilute-semidilute transition regime are becoming distinctly bent.

Part B of figure 5 demonstrates that the critical concentrations (expressed in units of $c^{*}$ ), dividing concentration regimes with an opposite sign of the birefringence signal, are the same for the different molecular weights (MW400, MW1132, also MW200 shows the same behaviour).

The concentration of about $20 c^{*}$ PSS $^{-}$at minimum ionic strength seems to be universally valid, also for other experiments described in the literature : Dalbiez et al. [38] performed electrophoretic measurements and found a critical concentration of $2.5 \times 10^{-2} \mathrm{mg} / \mathrm{ml}$ for MW780 $\left(=16 c^{*}\right)$. The scattering results of Kaji $e t$ al and the birefringence results of Krämer and Oppermann mentioned above establish nearly the same critical concentration. Nierlich et al. [5] report a change of the power law describing the concentration dependence of the persistence length and the radius of gyration, respectively, at a monomer concentration of about $0.14 \mathrm{M}$ of PSS with MW $=2.6 \times 10^{4} \mathrm{~g} / \mathrm{mol}\left(=21 c^{*}\right)$. Finally Drifford $e t$ al. [22] note an appearance of one diffusion coefficient below about $1.6 \mathrm{mg} / \mathrm{ml}$ of MW100 $\left(=18 c^{*}\right)$ and a second one above about $4.9 \mathrm{mg} / \mathrm{ml}\left(=53 c^{*}\right)$. The crossover concentration $c_{\mathrm{S}}^{*}$ calculated by Kaji (Eq. (5a)) coincides with our critical concentration exactly. Only data published recently by Oostwal and Odijk [39] show no critical concentration. Nevertheless, their establishing of the empirical quantity $\mathrm{X}$ in order to find a molecular weight independent quantity is not necessary: already their viscosity data of PSS31, PSS 88 and PSS 177 (without added salt) plotted versus the concentration in units of $c^{*}$ does not reveal any molecular weight dependence.

However, the second critical concentration of about $1000 \mathrm{c}^{*}$, also described by Krämer and Hoffmann [9], we observe in the electric birefringence has no equivalent to be found in other than birefringence experiments and remains somewhat mysteriously. One explanation could be a twisting of aromatic groups which could simulate a normal birefringence signal in spite of particles being perpendicularly oriented to the electric field. The fact that the molecular weight independent concentration where the change of the birefringence signal occurs has to be measured in units of $c^{*}$ and not in units of $\mathrm{mg} / \mathrm{ml}$ gives us the hint that still rodlike properties are important. Therefore changes of the molecular structure as twisting of aromatic groups are unlikely.

\section{Conclusions.}

Aqueous solutions of poly(styrenesulfonate) chains have been examined at minimum ionic strength (down to $10^{-6} \mathrm{M}$ ) by static light scattering (SLS) and electric birefringence experiments. The SLS experiments on aqueous solutions of $\mathrm{PSS}^{-}$show a single broad but well defined peak. The position $q_{\mathrm{m}}$ of this peak scales with concentration $c$ as $c^{1 / 3}$ for dilute solutions and like $c^{1 / 2}$ for semidilute ones. The position where the transition from the $c^{1 / 3}$ - to the $c^{12}$-law occurs depends only on the concentration measured in units of $c^{*}$ and no longer on the molecular weight and the contour length, respectively. This law is also valid for the results of SAXS- and SANS-measurements. Furthermore we were able to orient particles by small electric fields $\left(4 \times 10^{2} \mathrm{~V} / \mathrm{m} \leqslant E \leqslant 3 \times 10^{4} \mathrm{~V} / \mathrm{m}\right)$ either parallel or perpendicular to these fields, and we presented the relaxation times of the birefringence signals after switching off the electric field. It was revealed that dilute suspended $\mathrm{PSS}^{-}$chains behave like expanded rods which are nearly rigid in the case of MW200 and MW400 and show some « bending 
properties " in the case of long chains (MW1132). The scattering and the birefringence experiments have a critical particle concentration of $20 c^{*}$ in common. In the former experiment a change of the power law mentioned above, in the latter one a change of the sign of the birefringence signal occurs at $20 c^{*}$. This concentration seems to be universally valid in nearly all other comparable experiments. With increasing concentration a coiling of the chains occurs; nevertheless they remain fairly rigid. Above $1000 c^{*}$ no further coiling happens and the particles behave like rigid extended ones with an effective length which is about one third of the contour length. The meaning of a second critical concentration observed in the electric birefringence is still unknown.

\section{Acknowledgements.}

This work was financially supported by the Deutsche Forschungsgemeinschaft (SFB 306).

\section{References}

[1] Hagenbuchle M., Weyerich B., Deggelmann M., Graf C., Krause R., Maier E. E., Schulz S. F., Klein R., Weber R., Physica A 169 (1990) 532.

[2] Krause R., Maier E. E., Deggelmann M., Hagenbuchle M., Schulz S. F., Weber R., Physica A 160 (1989) 135.

[3] Drifford M., Dalbiez J. P., J. Phys. Chem. 88 (1984) 5368.

[4] Nierlich M., Williams C. E., Boue F., Cotton J. P., Daoud M., Farnoux B., Jannink G., Picot C., Moan M., Wolff C., Rinaudo M., de Gennes P. G., J. Phys. France 40 (1979) 701.

[5] Nierlich M., Boue F., Lapp A., Oberthür R., J. Phys. France 46 (1985) 649.

[6] Kaji K., Urakawa H., Kanaya T., Kitamaru R., J. Phys. France 49 (1988) 993.

[7] Kramer H., Deggelmann M.. Graf C., Hagenbuchle M., Johner C., Weber R., Macromolecules 25 (1992) 4325.

[8] Kramer H., Graf C., Hagenbüchle M., Johner C., Martin C., Schwind P., Weber R., J. Phys. II France 4 (1994) 1061.

[9] Krämer U., Hoffmann H., Macromolecules 24 (1991) 256.

[10] Degiorgio V., Mantegazza F., Piazza R., Europhys. Letters 15 (1991) 75.

[11] Oppermann W., Macromol. Chem. 189 (1987) 927.

[12] Krause R., PhD Thesis, Universität Konstanz (1991).

[13] Graf C., Deggelmann M., Hagenbüchle M., Kramer H., Krause R., Martın C., Weber R., J. Chem. Phys. 95 (1991) 6284.

[14] Nierlich M., Boue F., Lapp A., Oberthur R., J. Collotd Polymer Sci. 263 (1985) 955.

[15] Förster S., Schmidt M., Antoniettı M., Polymer 32 (1990) 781.

[16] Ise N., Okubo T., Kunugi S., Matsuoka H., Yamaoto K.. Ishii Y., J. Chem. Phys. 81 (1984) 3294.

[17] Odijk T.. Macromolecules 12 (1979) 688.

[18] de Gennes P. G., Pincus P., Velasco R. M., Brochard F., J. Phys. France 37 (1976) 1461.

[19] Koyama R., Macromolecules 19 (1986) 178.

[20] Degiorgio V., Bellini L., Piazza R., Mantegazza F., Phys Rev. Lett. 64 (1990) 1043.

[21] Oppermann W., Macromol. Chem. 189 (1987) 2125.

[22] Drifford M., Bellonı T., Dalbiez J. P., Chattopadhyay A. K., J. Collold Interface Sci. 105 (1985) 587.

[23] Newman H., Swinney H. L., Day L. A., J. Mol. Bıol. 116 (1977) 593.

[24] Yoshizaki T., Yamakawa H., J. Chem. Phys. 81 (1984) 982.

[25] Asai H., Watanabe N., Biopolymers 15 (1976) 383.

[26] Kramer H., Martin C., Weber R., Prog. Colloid Interface Sci. to be published.

[27] Hoffmann H., Krämer U., Thurn H., J. Phys. Chem. 94 (1989) 2027. 
[28] Cates M. E., J. Phys. II France 2 (1992) 1109.

[29] Odijk T., Laser Light Scattering in Biochemistry, S. E. Harding, D. B. Sattele, V. Bloomfield Eds., Roy. Soc. Chem., London (1992).

[30] Vink H., Polymer 33 (1992) 3711.

[31] Reddy M., Marinsky Y. A., J. Chem. Phys. 74 (1970) 3884.

[32] Fredericq E., Houssier C., Electric Dichroism and Electric Birefringence (Clarendon, Press Oxford UK, 1973).

[33] Deggelmann M., Palberg T., Hagenbüchle M., Maier E. E., Krause R., Graf C., Weber R., J. Colloid Interface Sci. 143 (1991) 318.

[34] Graf C., Kramer H., Deggelmann M., Hagenbüchle M., Johner C., Martin C., Weber R., J. Chem. Phys. 98 (1993) 4920.

[35] Oppermann W., Habilitation Thesis, Technische Universität Clausthal-Zellerfeld, Germany (1986).

[36] Stevens M. J., Kremer K., Macromolecules 26 (1993) 4717.

[37] Schulz S. F., PhD Thesis, Universität Konstanz (1989).

[38] Dalbiez J. P., Tabti K., Derian P. J., Drifford M., Rev. Phys. Appl. 22 (1987) 1013.

[39] Oostwald M., Odijk T., Macromolecules 26 (1993) 6489. 